\title{
Atención, experiencias y lugar de la familia en unidades de cuidado neonatal de Antioquia, Colombia
}

\section{Silvia María Castañeda Rivillas}

Magíster. Directora de la Facultad de Trabajo Social de la Universidad Pontificia Bolivariana.

Correo: silvia.castaneda@upb.edu.co

\section{Johanna Jazmín Zapata Posada}

Doctora. Profesora interna de la Facultad de Trabajo

Social de la Universidad Pontificia Bolivariana.

Correo:johanna.zapata@upb.edu.co

\section{Manuela Agudelo Peláez}

Trabajadora social.

Aprobado:

5 de julio de 2020

Recibido:

30 de marzo de 2020

DOI:

\section{Juliana Restrepo Valderrama}

Trabajadora social.

Correo: juliana.restrepova@upb.edu.co 


\section{Resumen}

Introducción: los recién nacidos de riesgo tienen derecho a estar con su familia e incluirla en la Unidad de Cuidado Neonatal (UCN), lo cual resulta beneficioso, esto implica innovar la atención en salud. Metodología: estudio de caso cualitativo que buscó reconocer la situación de las familias en tres UCN de Antioquia a través de entrevista semiestructurada a tres profesionales y a veintitrés familias. Resultados: las UCN promueven la inclusión de las familias, que perciben su experiencia como positiva, les brindan atención integral, con limitaciones. Conclusión: es necesario crear un modelo contextualizado para la participación de las familias en las UCN de Antioquia.

Palabras clave

Recién nacido, Familia, Cuidadores, Atención posnatal, Unidades de cuidado intensivo neonatal.

\section{Abstract}

Introduction: High-risk newborns have the right to be with their families and including the family in the neonatal care unit (NCU) is beneficial; this imply to innovate the health care. Methodology: Qualitative case study to recognize the situation of families at 3 NCUs in Antioquia through semi-structured interviews with 3 professionals and 23 families. Results: NCUs incorporate practices promoting the inclusion of families. The families consider that their experiences are positive. Comprehensive care is given, although there are some constraints. Conclusion: It is necessary to create a contextualized model for the family participation in NCUs of Antioquia.

Keywords

Newborn; Family; Caregivers; Postnatal Care; Intensive care units, neonatal. 


\section{Introducción}

Los procesos de gestación, nacimiento y los primeros días de vida del ser humano contienen una serie de factores que ponen en riesgo el desarrollo de algunos bebés, conocidos estos como recién nacidos de riesgo (RNR), entre los que se encuentran los prematuros, los de bajo peso, aquellos con malformaciones congénitas y los que nacen con complicaciones en el parto. Estos bebés presentan alguna anomalía, transitoria o definitiva, en su desarrollo motor, sensorial, cognitivo o conductual (Robles, Poo y Poch, 2008, p. 278), por tanto requieren ser internados en unidades de cuidado neonatal (UCN) donde se les brinda atención especializada con profesionales idóneos, espacios adecuados, equipos y procedimientos de alta complejidad (Riani, 2007, p. 138). Desde diferentes esferas del conocimiento, se reconoce el derecho de los RNR a estar con su familia y la importancia de esta en su cuidado como agente protagonista durante la atención en la UCN. Esto ha llevado al surgimiento de innovaciones en el enfoque de atención en estos servicios de salud, como el modelo de cuidado centrado en la familia (MCCF) presente en algunas UCN de Europa, Estados Unidos y América Latina. Según el Ministerio de Sanidad, Servicios Sociales e Igualdad (2014), este modelo en España promueve un cambio de actitud al reconocer a la familia como un agente prioritario en el proceso de hospitalización y recuperación del niño en cuanto a sus cuidados y toma de decisiones frente a estos (p. 47).

Diferentes estudios han encontrado que la implicación parental en el cuidado del RNR, aporta en su recuperación, mejora la calidad del vínculo afectivo, contribuye al aumento de la tasa de lactancia, al desarrollo psicomotor y neurosensorial y facilita los cuidados posteriores a la hospitalización pues reduce el reingreso a los servicios de salud (Ministerio de Sanidad, Servicios Sociales e Igualdad, 2014, p. 47).

Para el año 2016, el departamento de Antioquia contaba con once $\mathrm{UCN}^{1}$, la mayoría de ellas ubicadas en la ciudad de Medellín y el área metropolitana, lo que la hace un referente en la atención al RNR y la constituye en una ciudad receptora de estos pacientes y sus familias. En el año 2015 se diseñó la política pública departamental Buen Comienzo Antioquia como soporte

1 Dato construido por las autoras a partir de diversas fuentes disponibles a la fecha del estudio, debido a que no se encontró un informe oficial al respecto. 
institucional y estratégico para el pleno desarrollo de las capacidades de los niños y niñas desde la gestación y durante su primera infancia (Asamblea Departamental de Antioquia, 2015). Dentro de las líneas estratégicas de la política se encuentra la acogida de niños y niñas al nacer, con reconocimiento de su dignidad; uno de sus objetivos es:

Promover la implementación del programa de atención y seguimiento integral al recién nacido en situaciones de prematuridad, que manifiesta salud vulnerable o alteraciones en el desarrollo, enfocando al apoyo a la familia para proveer cuidados centrados en el desarrollo de niños y niñas y a dar soporte afectivo en un ambiente respetuoso (Asamblea Departamental de Antioquia, 2015, p. 7).

Si bien Antioquia cuenta con oferta institucional de atención al RNR y tiene política pública que promueve el apoyo a la familia, es necesario establecer protocolos y procedimientos para fortalecer el lugar de la familia en las UCN, a partir de un modelo que promueva y direccione su inclusión.

En este escenario surge el proyecto Cuidado centrado en la familia al recién nacido de riesgo atendido en unidades neonatales, el cual fue ejecutado entre los años 2015 y 2017, con la participación de diferentes instancias académicas de la Universidad Pontificia Bolivariana y con el propósito de aportar a la construcción de un modelo que permita, no solo mejorar las condiciones de vida para los RNR, sino también el fortalecimiento del vínculo familiar.

El proyecto se estructuró a través de tres componentes: i) estado del arte sobre el MCCF; ii) reconocimiento de la situación de las familias en las UCN de Antioquia; iii)caracterización de RNR atendidos en la Fundación Milagros de Vida. En el presente artículo se reportan los resultados del segundo componente, cuyo objetivo fue reconocer la situación de las familias en las UCN de Antioquia, respecto a la atención, las experiencias y el lugar que se les asigna para el cuidado del RNC. 


\section{Metodología}

La investigación tuvo un diseño cualitativo y su modalidad es estudio de caso utilizando estrategias metodológicas de corte etnográfico y narrativo (Galeano, 2012; Hernández, Fernández y Baptista, 2014; Sabino, 2014), en las que la interacción y el lenguaje de las personas cobran gran importancia, pues es a través de ellas que se materializan las prácticas sociales (Galeano, 2012).

El estudio se realizó durante el año 2016 en tres UCN de Antioquia que aceptaron participar luego de que sus respectivos comités de ética aprobaran el protocolo de la investigación. Estas instituciones fueron: Unidad Materno Infantil Nuestra Señora de la Candelaria de la Clínica Universitaria Bolivariana en Medellín, Unidad de Cuidados Intensivos Neonatal del Hospital Manuel Uribe Ángel en Envigado y Unidad Materno Infantil Fundación SOMA en Chigorodó.

Los participantes del estudio fueron tres profesionales del equipo médico encargados de cada UCN y veintitrés familiares (padres, madres y abuelos), que tenían sus RNR hospitalizados. La participación fue voluntaria y con firma del consentimiento informado para cumplir con los criterios de inclusión.

Se utilizó la entrevista semiestructurada para acceder a la información: tres individuales (con cada profesional médico) y tres grupales (familias del RNR). Las entrevistas fueron grabadas en formato de audio, luego se transcribieron y codificaron para clasificar los datos según la matriz de análisis categorial. Para el análisis de la información se construyeron los memos analíticos a partir de la lectura intratextual e intertextual, los cuales fueron el insumo principal para la consolidación de los resultados obtenidos. Cabe resaltar que los testimonios se presentan con códigos de la siguiente manera: EG: entrevista grupal con familias; EI: entrevista individual con profesional médico; y seguido de UCN\# que corresponde al número asignado a cada una de las tres unidades.

La unidad de análisis del estudio fue el cuidado centrado en la familia que se desarrolló a partir de categorías y subcategorías, a través de las cuales se buscó caracterizar la atención ofrecida a las familias, explorar acerca de su experiencia en la UCN e identificar el lugar que se les asigna en el cuidado a los RNR. Las categorías y subcategorías se distribuyeron de 
la siguiente manera: (a) Atención a las familias -equipo médico, equipo psicosocial, espacios y adecuación, aspectos diferenciadores, programas dirigidos a las familias, recursos disponibles para la atención a las familias, tipo de acompañamiento, satisfacción de las necesidades de las familias, necesidades y limitaciones-; (b) Experiencias de las familias -estrés y tensiones, adaptación y aprendizaje, percepción de la experiencia-; y (c) Lugar de la familia -participación en el cuidado, restricciones para la participación, percepción del equipo médico sobre la familia-.

\section{Resultados}

- Categoría 1: atención a las familias

Las UCN brindan atención de calidad a los RNR a través de alta tecnología y protocolos estandarizados, atención individualizada y acompañamiento médico durante veinticuatro horas, además que cuentan con los niveles de complejidad de cuidado intensivo, cuidado intermedio y cuidado básico.

Las tres UCN disponen de un equipo médico conformado por profesionales de la salud, y un personal de enfermería que tiene mayor contacto con las familias. Las familias manifiestan que, en general, el equipo médico es idóneo, aunque desearían recibir una mejor atención por parte de algunos profesionales. Al respecto, se evidenciaron esfuerzos por humanizar la atención por parte de las UCN a través de sensibilización y capacitación al personal, teniendo en cuenta que las diversas necesidades de las familias requieren de una intervención integral, que trasciende los servicios de salud ofrecidos al RNR; "aunque el acompañamiento de ellas [las enfermeras] es constante, creo que les falta un poquito más de delicadeza y de cuidados con la atención en la mamá” (EGUCN1).

Solo dos UCN cuentan con equipo psicosocial que trabaja en articulación con el equipo médico. En la tercera UCN, el equipo médico es quien acompaña a las familias y reconoce la necesidad de incluir profesionales sociales: "necesitamos obviamente que se le haga un abordaje más completo y más detallado a la familia, es decir, necesitamos trabajadora social, necesitamos 
la psicóloga" (EIUCN3). Ambos equipos destacan que el área psicosocial es importante y necesita ser fortalecida.

Por otra parte, los profesionales buscan establecer alianzas y gestionar recursos para satisfacer algunas necesidades de las familias que no estén al alcance de la UCN y que permitan una atención más integral. Entre los recursos y programas disponibles para las familias, se encuentran el apoyo emocional y espiritual, terapia familiar, atención en crisis, orientación y remisión según la problemática familiar, asesorías frente a derechos y deberes, apoyo económico para transporte, alimentación, dotación de implementos básicos para los RNR y albergue para familias que residen en lugares alejados de las UCN.

Las UNC también cuentan con actividades de formación sobre diversas temáticas para empoderar a las familias en el cuidado al $\mathrm{RNR}$. Al respecto, las familias y el equipo profesional manifiestan la necesidad de incluir otros temas y metodologías acordes a su realidad, como es el caso de un equipo médico que respete las costumbres familiares, a la vez que corrija prácticas que puedan afectar al RNR: "no tiene que estar por encima del bienestar del paciente (...). Pero, reiterando, sí tenemos siempre en cuenta los puntos de vista de ellos [padres]" (EIUCN3).

La mayoría de las familias manifiestan que el acceso a la información sobre el estado de salud del RNR es suficiente, aunque algunas quisieran que el personal médico utilizara un lenguaje que puedan comprender: "le hablan a uno como en el hablado de ellos y uno no les entiende [refiriéndose al equipo médico]" (EGUCN2).

En cuanto a la infraestructura, las familias y el equipo médico de dos UCN perciben que los espacios son reducidos y no permiten la privacidad, o no cuentan con las condiciones necesarias para la atención a los RNR y sus familias: "falta mejorar la infraestructura" (EIUCN2); "en espacios, todas las unidades ustedes van a encontrar ustedes que están súper limitadas" (EIUCN1). En la tercera UCN sí contaban con una sala privada y cómoda. 
- Categoría 2: experiencias de las familias

Para las familias, la experiencia dentro de la UCN está acompañada de sentimientos ambiguos: "tener que verlos todos chiquiticos con todos esos aparatos encima es difícil. Uno quiere saber por qué, qué le están haciendo" (EGUCN2).

La incertidumbre y la tristeza que genera el proceso en la UCN se contrarrestan con la seguridad y tranquilidad que sienten las familias por el apoyo y el acompañamiento del equipo profesional, con la recuperación del RNR y el apoyo de otras familias, factores que posibilitan la adaptación y la resignificación de su experiencia. Esto, sumado a la satisfacción por la superación y los aprendizajes adquiridos, les permiten percibir que su experiencia es positiva a pesar de las adversidades: "es una experiencia dura, muy maluco tener que dejarlos acá y todo eso, pero uno sabe en el fondo que es lo mejor" (EGUCN1).

- Categoría 3: lugar de la familia

Tanto el equipo profesional como las familias consideran que el papel de la familia es fundamental, es por esto que participan y comparten las tareas de la atención, el cuidado y la recuperación de los RNR.

No obstante, existen ciertas restricciones para la participación, teniendo en cuenta el nivel de complejidad en la atención y también el bienestar del RNR. Por ejemplo, en las UCN consideran que el acompañamiento de los padres es suficiente y, teniendo en cuenta el espacio limitado y la salud y seguridad del RNR, solo permiten el ingreso de una persona, privilegiando además, el acompañamiento de la madre por la lactancia materna. En una UCN permiten el ingreso de otros allegados cuando se demuestra un beneficio real para el $\mathrm{RNR}$. Al respecto, las familias quisieran incluir a otros familiares como hermanos o abuelos con el argumento de que el cuidado es una tarea agotadora; en ocasiones tienen algún impedimento por cuestiones de salud o asuntos familiares, y hay madres que no cuentan con el apoyo de su pareja; sin embargo, reconocen que el espacio es insuficiente y que puede ser riesgoso para el RNR. 
Por otra parte, existen factores externos a la UCN que limitan la participación de las familias, como las dificultades socioeconómicas, la lejanía del lugar de residencia, las responsabilidades laborales, el no contar con la licencia de maternidad legal en Colombia, el poco compromiso de algunas familias y, finalmente, aspectos culturales como la diferencia de lenguajes (como el caso de poblaciones indígenas) y la práctica de la dieta durante cuarenta días posparto, que implica que las madres estén en reposo absoluto en sus hogares.

\section{Discusión}

Los resultados de este estudio permiten reconocer la situación de las familias en tres UCN de Antioquia, lo cual es un avance significativo en la comprensión de esta realidad social prioritaria. Este estudio puede ser replicable en otras UCN en contextos socioeconómicos similares en los ámbitos nacional e internacional. En cuanto a las limitaciones de la investigación, se destacan la dificultad para captar la participación de las UCN debido a las dinámicas propias del sistema de salud, a restricciones de tipo institucional y a la disponibilidad del equipo médico.

En consonancia con la Organización Mundial de la Salud [OMS] (2010), durante el proceso de recuperación del RNR, este tiene derecho a estar acompañado por su familia todo el tiempo. En las UCN, se encontró, de un lado, que cada RNR es atendido de manera personalizada y permanente por el equipo médico, teniendo presente que estos pacientes requieren atención individualizada según sus necesidades (Ministerio de Sanidad, Servicios Sociales e Igualdad, 2014, p.14). De otro lado, en las UCN participantes se permite la presencia permanente de la familia, con el fin de facilitar el cuidado, fomentar el vínculo con el RNR y favorecer la unión familiar (Cañas y Rodríguez, 2014, pp. 343-344, 347-348; Ministerio de Sanidad, Servicios Sociales e Igualdad, 2014, p. 47).

Desde una visión tradicional del cuidado, se asigna a la madre un lugar protagónico que se refuerza por la importancia de la lactancia materna, brindándole prioridad a su presencia dentro de las UCN (OMS, 2010, p. 28; Villamizar, Vargas y Rueda, 2014, p. 787; Villamizar, Vargas y Rueda, 2017, p. 44); por su parte, el padre tiene menor participación y se le demandan 
asuntos de proveeduría económica, lo que puede limitar su presencia activa dentro de la UCN. Sobre la inclusión de otros familiares, se identificó que es permitido en las UCN solo cuando se demuestra un beneficio real para el RNR, lo cual perfila un reto para el sistema de salud, en tanto familiares como hermanos y abuelos, en general, suelen ser un soporte importante para las familias (Ministerio de Sanidad, Servicios Sociales e Igualdad, 2014, p. 15). La mayor participación del padre y de otros familiares, demandan un cambio de paradigma desde una perspectiva integral del cuidado (Cañas y Rodríguez, 2014, p. 342).

Algunos estudios coinciden en afirmar que cuando se hospitaliza al RNR, también "se hospitaliza" a su familia, lo que implica cambiar el paradigma de atención (Mendes y Martins, 2011, p. 339), para ello es necesario trascender la visión tradicional de los servicios de salud e incluir reformas locativas y tecnológicas, acciones de comunicación, formación y apoyo psicosocial para la familia del RNR, lo cual inicia con el reconocimiento de las particularidades de cada caso, el respeto a las características socioculturales, a la dignidad y a la privacidad, entre otras (Mendes y Martins, 2011, p. 337; Ministerio de Sanidad, Servicios Sociales e Igualdad, 2014, pp. 11-21; Tobo, Betancur y De la Cruz, 2017, p. 172).

Otros estudios han evidenciado que la relación entre el equipo profesional y las familias en UCN requiere respeto, comprensión y empatía (Patiño y Reixach, 2009, p. 91) y que, para afianzar esta relación, es fundamental la confianza (Cañas y Rodríguez, 2014, pp. 346-347) y el trabajo conjunto (Balbino, Balieiro y Mandetta, 2016, p. 2). El equipo médico debe estar dispuesto a incluir a las familias en el cuidado por varias razones: porque estas requieren sentirse útiles (Patiño y Reixach, 2009, p. 94), porque perciben que su participación es inherente a su función como cuidadoras y porque su presencia es de apoyo para el equipo (Mendes y Martins, 2011, pp. 339, 342 ), dado que algunas de sus funciones son irreemplazables (Tobo et al., 2017, p. 172; Unicef, 2013, p. 9). En esta línea, la investigación encontró que las familias desempeñan funciones básicas de supervisión, alimentación, higiene, acompañamiento físico y afectivo; además se reconoce que las UCN promueven el trabajo colaborativo entre las familias y el equipo médico para fortalecer el cuidado al RNR, y entre las mismas familias como apoyo mutuo. 
Una de las necesidades principales en las UCN es la comunicación (Cañas y Rodríguez, 2014, pp. 344, 346, 348; Patiño y Reixach, 2009, p. 94) debido a que las familias están en el derecho a conocer todo lo que acontece y de esta forma participar en el cuidado y en las decisiones (Unicef, 2013, p. 9). El equipo médico, brinda información permanente sobre el funcionamiento de la UCN, los derechos y deberes, y el proceso del RNR (Ministerio de Sanidad, Servicios Sociales e Igualdad, 2014, pp. 12, 51-52, 54, 86) e incluyen a la familia en algunas decisiones cuando se considera importante su opinión, sin embargo, la familia demanda mayor involucramiento e información amplia, oportuna y comprensible. Otras necesidades, como el apoyo emocional (Balbino et al., 2016, p. 2) y espiritual (Cañas y Rodríguez, 2014, pp. 346-347) son atendidas a través del apoyo psicosocial.

Respecto al rol de las familias como cuidadoras, es necesario que en las UCN se fomente el aprendizaje sobre los cuidados al RNR a través de actividades formativas (Ministerio de Sanidad, Servicios Sociales e Igualdad, 2014, p. 49). Al respecto, se encontró que ofrecen capacitaciones en temas como alimentación, higiene, salud y vínculo afectivo, con el reto de incluir nuevas estrategias metodológicas y promover que los aprendizajes adquiridos sean puestos en práctica en las tareas diarias de cuidado al RNR (Ministerio de Sanidad, Servicios Sociales e Igualdad, 2014, pp. 11-12, 49) en la UCN y en sus hogares (Mendes y Martins, 2011, p. 341; Patiño y Reixach, 2009, p. 93). Otro reto para las UCN es fortalecer el espacio físico, debido a que es reducido, no permite la privacidad y no cuenta con la dotación suficiente. Es fundamental que sean entornos confortables destinados a los padres y a otros integrantes de las familias para acompañar la hospitalización (Cañas y Rodríguez, 2014, pp. 339, 345, 348; Ministerio de Sanidad, Servicios Sociales e Igualdad, 2014, pp. 109-111).

Cabe resaltar que en las UCN, además de implementar acciones para las familias, también consideran necesario brindar apoyo a los profesionales, lo cual está en consonancia con los hallazgos de otras investigaciones (Mendes y Martins, 2011, p. 342); así, se encuentra que, a pesar de tener algunas iniciativas al respecto, como sensibilización en atención humanizada, es un aspecto que se debe fortalecer, especialmente en el personal de enfermería que es quien está en mayor contacto con las familias y por ende ocupan un papel fundamental (Cañas y Rodríguez, 2014, p. 347). 


\section{Conclusiones}

Las UCN promueven el abordaje integral de las familias soportadas en el equipo profesional con el que cuenten, sea médico o psicosocial, y se evidencian esfuerzos por humanizar la atención a las familias y a los RNR. Las UCN incluyen dentro de sus acciones programas de formación, apoyo legal y económico, los cuales aportan a la satisfacción de sus necesidades y al cumplimiento de sus derechos. Los aspectos que limitan la atención a las familias están representados en la necesidad de fortalecer el equipo psicosocial, la insuficiente disponibilidad de recursos económicos y las limitaciones en la infraestructura física.

Aunque la experiencia de cada familia es singular, existen expresiones emocionales comunes. De un lado manifiestan temor e incertidumbre frente a la crisis que vivencian $y$, del otro, se sienten esperanzadas frente al proceso de recuperación del RNR, aprecian el acompañamiento que reciben del equipo profesional y la solidaridad de otras familias en su misma situación. En general, las familias resignifican su experiencia y resaltan el logro de aprendizajes significativos.

Se reconoce que, tanto las familias como el equipo profesional, consideran que el lugar de la familia es fundamental en el proceso de atención al RNR. El equipo profesional está interesado en el compromiso y la participación de las familias en los cuidados de los RNR, y por ello promueven y motivan su inclusión, teniendo en cuenta las restricciones que se puedan generar de acuerdo al nivel de complejidad en la atención y priorizando en todo momento el bienestar del RNR.

Promover la participación de la familia en el cuidado del RNR atendido en UCN es un pilar primordial para su recuperación. En Colombia, particularmente en el departamento de Antioquia, se cuenta con una oferta institucional de atención al RNR y con una política pública que promueve la participación de la familia en sus cuidados. Los resultados de este estudio referidos al reconocimiento de la realidad de las familias, la atención que reciben, la experiencia y su lugar en el cuidado del RNR, invitan a articular las acciones desarrollas desde las UCN, en aras de apropiar un modelo de cuidado contextualizado centrado en la familia. 


\title{
Financiamiento
}

\author{
Ninguno.
}

\section{Conflictos de intereses}

Los autores declaran no tener ningún conflicto de intereses.

\section{Referencias}

Asamblea Departamental de Antioquia. (2015, 21 de diciembre). Ordenanza 26. Por medio de la cual se crea la política pública departamental Buen Comienzo Antioquia, para el pleno desarrollo de las capacidades de los niños y niñas desde la gestación y durante su primera infancia en el departamento de Antioquia. http://www.asambleadeantioquia.gov. co/2016/index.php?option $=$ com_jdownloads\&task $=$ download.send\&id $=2157 \&$ catid $=$ $10 \& \mathrm{~m}=0 \&$ Itemid $=792$

Balbino, F., Balieiro, M. y Mandetta, M. (2016). Measurement of Family-centered care perception and parental stress in a neonatal unit. Revista Latino-Americana de Enfermagem, 24, 1-9. http://dx.doi.org/10.1590/1518-8345.0710.2753

Cañas-Lopera, E. M. y Rodríguez-Holguín, Y. A. (2014). La experiencia del padre durante la hospitalización de su hijo recién nacido. Pretérmino extremo. Aquichan, 14(3), 336-350. https://bit.ly/3iVylyT

Fondo de las Naciones Unidas para la Infancia. Unicef. (2013). Decálogo de los derechos del prematuro y su familia. https://uni.cf/3kD7BlS

Galeano, M. E. (2012). Estrategias de investigación social cualitativa. El giro en la mirada. La Carreta Editores.

Hernández, R., Fernández, C. y Baptista, P. (2014). Metodología de la investigación. McGraw-Hill.

Mendes, M. G. y Martins, M. M. (2011). La experiencia de las madres en la participación del cuidado de sus hijos hospitalizados. Enfermería Clínica, 21(6), 338-343. http://dx.doi. org/10.1016/j.enfcli.2011.07.009

Ministerio de Sanidad, Servicios Sociales e Igualdad. (2014). Unidades de neonatología: estándares y recomendaciones de calidad. Ministerio de Sanidad, Servicios Sociales e Igualdad. https://bit.ly/33U5Ytl 
Organización Mundial de la Salud. OMS. (2010). Trabajando con individuos, familias y comunidades para mejorar la salud materna y neonatal. http://apps.who.int/iris/bitstream/ handle/10665/70173/WHO_MPS_09.06_spa.pdf? sequence $=1$

Patiño, J. y Reixach, M. (2009). ¿Qué tipo de ayuda demandan los padres de recién nacidos hospitalizados en cuidados intensivos neonatales? Enfermería Clínica, 19(2), 90-94. http://dx.doi.org/10.1016/j.enfcli.2008.11.001

Robles, M. C., Poo, M. P. y Poch, M. L. (2008). Atención temprana: recursos y pautas de intervención en niños de riesgo o con retrasos del desarrollo. Asociación Española de Pediatría, (37), 278-285. https://bit.ly/3kFFu5z

Riani, N. (2007). Momentos clave para humanizar el paso por la unidad de cuidados intensivos neonatal. Persona y Bioética, 11(29), 138-145. http://www.scielo.org.co/pdf/pebi/ v11n2/v11n2a04.pdf

Sabino, C. (2014). El proceso de investigación. Episteme.

Tobo, N., Betancur, P. y De la Cruz, C. (2017). Estímulos, afrontamiento y adaptación en padres de recién nacidos hospitalizados en unidades de cuidado intensivo neonatal. Investigación en Enfermería: Imagen y Desarrollo, 19(2), 161-175. https://doi.org/10.11144/ Javeriana.ie19-2.eaap

Villamizar, B., Vargas, C. y Rueda, E. (2014). Madres descubriendo el amor incondicional en el proceso adaptativo de hospitalización de su bebé prematuro. Cuidarte, 5(2), 782-791. http://dx.doi.org/10.15649/cuidarte.v5i2.93

Villamizar, B., Vargas, C. y Rueda, E. (2017). Actitudes de madres adultas y adolescentes hacia la crianza de bebés prematuros. Investigación en Enfermería: Imagen y Desarrollo, 19(2), 41-52. http://dx.doi.org/10.11144/Javeriana.ie19-2.amaa 